\title{
Генераторы изображений для безмасковой литографии
}

\author{
С. Аваков д. т. н. ${ }^{1}$, В. Плебанович к. т. н. ${ }^{2}$, А. Лапко
}

УДК 621.38 | ВАК 05.27.06

\begin{abstract}
Многие годы технологи фотолитографии работают на традиционных установках совмещения и экспонирования - проекционной или контактной печати. Но за последнее время инженеры-конструкторы создали такие лазерные генераторы изображений (ГИ), которые по точности переноса топологии превзошли все ранее известные способы. Многолучевые лазерные генераторы изображений позволяют на практике внедрять в серийное производство безмасковую литографию.
\end{abstract}

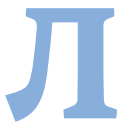
азерные генераторы не могут похвастаться разрешением лучше 200 нм, но зато они позволяют исключить затраты на производство фотошаблонов, для них нет ограничений на размеры кристалла, и реализация multi project не требует дополнительных затрат, их скорость печати высока по сравнению с генераторами E-bеam. Все это открывает новые возможности для разработчиков микросхем и их производства.

Если вам не удается вписать все функции устройства в кристалл типовых размеров вы можете кристалл увеличить до нужных размеров и создать полнофункциональное устройство на уровне кристалла, а не платы. Это позволит не только уменьшить массогабаритные характеристики, но и повысить надежность. Возможность менять топологию рядом расположенных кристаллов в одном технологическом цикле позволяет создавать изделия, близкие по техническим параметрам, но разные по функциональному применению. Возможности конструктора в данном случае становятся безграничными [1].

Но как доказать, что генераторы изображений обеспечивают качество переноса изображения лучше, чем повсеместно используемые установки проекционной печати, которые на сегодняшний день являются эталоном технологического оборудования для фотолитографии. Основным критерием оценки качества является расчет индексов воспроизводимости.

Индекс воспроизводимости - это коэффициент, показывающий во сколько раз граница допуска на параметр, установленная в конструкторской документации, больше, чем фактическое среднеквадратичное отклонение параметра, обеспечиваемое технологией. Графическое представление распределения технологического параметра при разных

ОАО «КБТЭМ-ОМО», Республика Беларусь, г. Минск

ASM@kbtem-omo.by.

ОАО «КБТЭМ-ОМО», Республика Беларусь, г. Минск,

vpleba@kbtem-omo.by. значениях среднеквадратичного распределения (б) представлено на рис. 1. Если допуск на параметр измерять в величинах среднеквадратичного отклонения параметра, то чем меньше значение среднеквадратичного распределения, тем больше количество его значений может находиться в его пределах.

Из теории статистического анализа известно, что если величина допуска для параметра равняется двум среднеквадратичным отклонениям, то в этот допуск (2б) попадет только 68,3\% всех значений выборки, а 31,7\% значений не будут соответствовать требованиям конструкции и, следовательно, должны быть забракованы. Результаты расчетов процента выхода

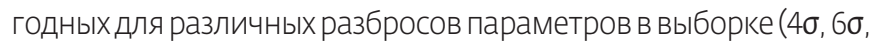
$8 \sigma, 10 \sigma)$ представлены в табл. 1. В зависимости от количества последовательных операций с данными значениями разброса можно рассчитать результирующий процент выхода для технологического маршрута. Если взять простой КМОП-маршрут

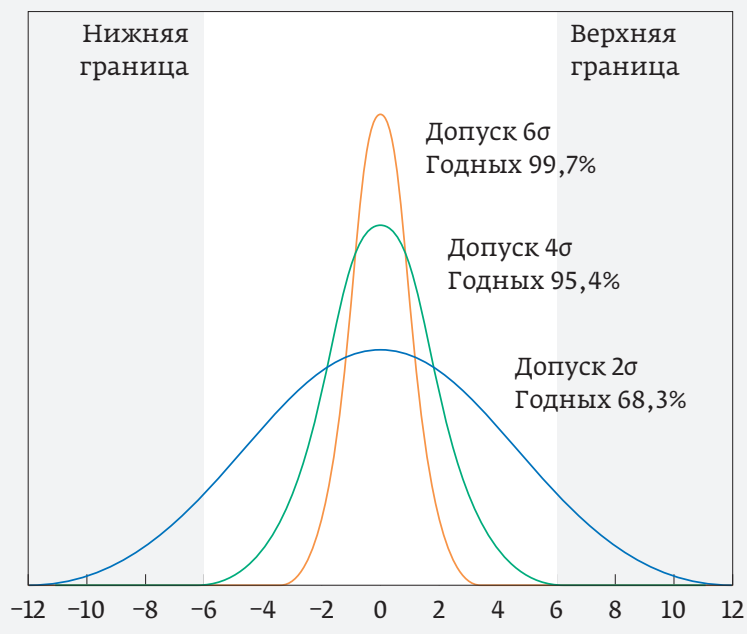

Рис. 1. Нормальное распределение параметра в рамках допуска для различных значений среднеквадратичного распределения 
для технологических норм 2-3 мкм, то он состоит из 100 технологических операций. Если унас для каждой операции на маршруте разброс параметров будет вкладываться в 4б от допуска, то окончательный процент выхода будет составлять 0,9\%, что неприемлемо. Чем меньше разброс параметров, тем меньше значение среднеквадратичного распределения, тем большее количество этих значений может вместиться в допуск на параметр, следовательно, выше будет процент выхода годных. Исходя из приведенных аргументов допуск на параметр является важнейшейхарактеристикой в оценке качественныхпоказателей сравниваемых единиц оборудования

Сравним лазерные генераторы изображений производства ОАО «КБТЭМ-ОМО» и степперы японской фирмы Nikon. Генератор изображения ЭМ-5589 предназначен для безмасковой литографии с технологическими нормами 0,6 мкм, а генератор ЭМ-5289Б со степперами - для технологических норм 0,35 мкм. Для данных технологических норм японская фирма Nikon была лидером по производству степперов, поэтому выберем установку NSR2005і8А для нормы 0,5 мкм и NSR2205i14E2 - для норм 0,35 мкм.

Если сравнить параметры генератора изображений и степпера аналогичного уровня технологии (табл. 2, 3), можно увидеть, что по основным параметрам, которые определяют
Таблица 1. Результаты расчета процента выхода для выборок с различным разбросом параметров и количества операций

\begin{tabular}{lllll} 
Допуск/ & 4 & 6 & 8 & 10 \\
Ср или Срк & 0,67 & 1,0 & 1,33 & 1,67 \\
\hline $\begin{array}{l}\text { \%лластин в допуске, } \\
\text { 1 операция на маршруте }\end{array}$ & 95,4 & 99,7 & 99,97 & 99,99 \\
\hline $\begin{array}{l}\text { \% пластин в допуске, } \\
\mathbf{1 0 ~ о п е р а ц и и ̆ ~ н а ~ м а р ш р у т е ~}\end{array}$ & 62,4 & 97 & 99,7 & 99,97 \\
\hline $\begin{array}{l}\text { \% пластин в допуске, } \\
\mathbf{1 0 0 ~ о п е р а ц и и ̆ ~ н а ~ м а р ш р у т е ~}\end{array}$ & 0,9 & 74 & 97 & 99,7 \\
\hline Оценка & Неуд. & Уд. & Хор. & Отлично \\
\hline
\end{tabular}

точность передачи топологии (точность расположения элемента, нелинейность элемента, воспроизводимость критического размера), генератор изображений ничем не уступает степперу. При этом нужно обратить внимание на то, что параметры степпера даются без учета точности изготовления шаблона. Величина точности изготовления фотошаблона соответствует параметрам генератора изображений, что с учетом масштаба экспонирования степпера (5:1) приведет к росту погрешности еще на 20\%. Если технические параметры генератора изображений и степпера сравнить с требованиями технологии для этих норм, то мы получим:

- при технологии 0,35 мкм (см. табл. 2) для генератора изображения индексы воспроизводимости лежат

Таблица 2. Сравнение основных параметров степпера и генератора изображений для технологии 0,35 мкм

\begin{tabular}{|c|c|c|c|c|c|c|}
\hline Параметры & $\begin{array}{l}\text { Tребования } \\
\text { ITRS }\end{array}$ & \multicolumn{2}{|c|}{$\begin{array}{c}\text { Генератор } \\
\text { изображений }\end{array}$} & Степпер & \multicolumn{2}{|c|}{$\begin{array}{l}\text { Степпер + } \\
\text { шаблон }\end{array}$} \\
\hline Наименование установки & & \multicolumn{2}{|c|}{ ЭМ-5289Б } & $\begin{array}{l}\text { Nikon } \\
\text { NSR2205i14E2 }\end{array}$ & \multicolumn{2}{|c|}{$\begin{array}{l}\text { Nikon } \\
\text { NSR2205i14E2 }\end{array}$} \\
\hline Проектная норма, мкм & 0,35 & \multicolumn{2}{|c|}{0,35} & 0,35 & \multicolumn{2}{|c|}{0,35} \\
\hline Поле экспонирования, мм & & \multicolumn{2}{|c|}{$215 \times 215$} & $22 \times 22$ & \multicolumn{2}{|c|}{$22 \times 22$} \\
\hline NA & & \multicolumn{2}{|c|}{0,78} & $0,5-0,63$ & \multicolumn{2}{|c|}{$0,5-0,63$} \\
\hline $\begin{array}{l}\text { Точность расположения элемента, нм } \\
\text { Lens Distortion, nm }\end{array}$ & \pm 80 & \pm 40 & $6,0 \sigma$ & \pm 35 & \pm 43 & $5,6 \sigma$ \\
\hline $\begin{array}{l}\text { Нелинейность элемента, нм } \\
\text { Resolution linearity }\end{array}$ & \pm 50 & \pm 30 & $5,0 \sigma$ & \pm 35 & \pm 41 & $3,7 \sigma$ \\
\hline $\begin{array}{l}\text { Воспроизводимость критического размера, нм } \\
\text { Isolated / Dense lines bias uniformity, nm }\end{array}$ & \pm 70 & \pm 25 & $8,4 \sigma$ & \pm 35 & \pm 40 & $5,3 \sigma$ \\
\hline $\begin{array}{l}\text { Точность совмещения с предыдущим слоем, нм } \\
\text { Total Overlay (Overlay + Stepping Precision), nm }\end{array}$ & \pm 110 & \pm 50 & $6,6 \sigma$ & \pm 75 & \pm 85 & $3,9 \sigma$ \\
\hline Наклон $\Phi / p$ & & & & $81^{\circ}$ & & \\
\hline Resist Slope & & & & $55 \%$ & & \\
\hline
\end{tabular}


Таблица 3. Сравнение основных параметров степпера и генератора изображений для технологии 0,6 мкм

\begin{tabular}{|c|c|c|c|c|c|c|}
\hline Параметры & $\begin{array}{l}\text { Требования } \\
\text { технологии }\end{array}$ & \multicolumn{2}{|c|}{$\begin{array}{c}\text { Генератор } \\
\text { изображений }\end{array}$} & Степпер & \multicolumn{2}{|c|}{$\begin{array}{c}\text { Степпер + } \\
\text { шаблон }\end{array}$} \\
\hline Наименование установки & & \multicolumn{2}{|c|}{$\ni M-5589$} & $\begin{array}{l}\text { Nikon } \\
\text { NSR2005i8A }\end{array}$ & \multicolumn{2}{|c|}{$\begin{array}{l}\text { Nikon } \\
\text { NSR2005i8A }\end{array}$} \\
\hline Проектная норма, мкм & 0,6 & \multicolumn{2}{|c|}{0,6} & 0,5 & \multicolumn{2}{|c|}{0,5} \\
\hline Поле экспонирования, мм & & \multicolumn{2}{|c|}{$215 \times 215$} & $20 \times 20$ & \multicolumn{2}{|c|}{$20 \times 20$} \\
\hline NA & & \multicolumn{2}{|c|}{0,6} & 0,5 & \multicolumn{2}{|c|}{0,5} \\
\hline $\begin{array}{l}\text { Точность расположения элемента, нм } \\
\text { Lens Distortion, nm }\end{array}$ & \pm 150 & \pm 60 & $7,5 \sigma$ & \pm 70 & \pm 82 & $5,5 \sigma$ \\
\hline $\begin{array}{l}\text { Нелинейность элемента, нм } \\
\text { Resolution linearity }\end{array}$ & \pm 90 & \pm 40 & $6,8 \sigma$ & \pm 50 & \pm 58 & $4,6 \sigma$ \\
\hline $\begin{array}{l}\text { Воспроизводимость критического размера, нм } \\
\text { Isolated / Dense lines bias uniformity, nm }\end{array}$ & \pm 120 & \pm 40 & $9 \sigma$ & \pm 50 & \pm 58 & $4,6 \sigma$ \\
\hline $\begin{array}{l}\text { Точность совмещения с предыдущим слоем, нм } \\
\text { Total Overlay (Overlay + Stepping Precision), nm }\end{array}$ & \pm 150 & \pm 70 & $6,4 \sigma$ & \pm 180 & \pm 194 & $2,3 \sigma$ \\
\hline Наклон $\phi / p$ & & & & $81^{\circ}$ & & \\
\hline Resist Slope & & & & $55 \%$ & & \\
\hline
\end{tabular}

в диапазоне от 5,0б до 8,40, для техже параметров степпера индексы воспроизводимости будут составлять от 3,7б до 5,6б, что значительно ниже;

- притехнологии 0,6 мкм (см. табл. 3)для генератора изображения индексы воспроизводимости в диапазоне от 6,4б до 9,0б, для тех же параметров степпера индексы

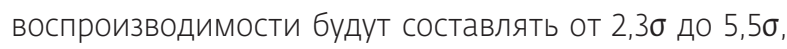
что значительно ниже.

По точности совмещения топологии степпер существенно уступает лазерному генератору изображений, так как суммарная погрешность степпера зависит от точности определения координат меток совмещения (что соизмеримо с точностью генератора) и точности перемещения между модулями экспонирования. В результате генератор переносит изображение второго слоя в 1,5-2 раза точнее.

Вторым важным преимуществом генератора изображений является поле экспонирования. Оно сегодня соответствует размеру 215 × 215 мм, хотя может быть сделано и больше по требованию заказчика. Размер поля экспонирования дает возможность не только рисовать кристаллы больших размеров, что востребовано при создании системы на кристалле (СНК), мультиплексоров, ПЗС-матриц, но и предлагать оптимальные объемы поставок различных зашивок БМК, плИС, пзУ и реализовывать multi project при разработке новых конструкций ИМС или при оказании услуг по фаундри. Одной пластиной, экспонируемой на генераторе изображений, вы можете заменить целую опытную партию изделий [2].

За всехорошее приходится расплачиваться временем экспонирования. Сегодня оно составляет около одного часа на пластину при однопроходном экспонировании, соответственно для получения качественного изображения требуется два прохода, что приведет к увеличению суммарного времени. Но техника не стоит на месте, появляются новые быстродействующие электрооптические, акустооптические, МЭМС-системы контроля и управления лучом лазера, что в итоге приводит к сокращению времени экспонирования.

Некоторые специалисты не видят проблем в длительных сроках выполнения операций ионной имплантации или термодиффузионных процессов, но при этом считают, что увеличенное время экспонирования может привести к росту дефектов на пластине при экспонировании при безмасковой литографии. Такие предположения не имеют под собой никаких оснований. Генератор состоит из узлов, которые не генерируют дефекты: стол перемещается на воздушной подушке, управление лучом лазера проводят электрооптические преобразователи. Запыленность внутри установки определяется классом чистоты помещения, в котором он расположен. Небольшие размеры оптомеханического устройства (ОМУ), полная его автономность позволяют создать такой уровень чистоты помещения, который обеспечит необходимые условия для бездефектной фотолитографии.

Сразу хочу предупредить читателей, что не все лазерные генераторы изображений обладают такими параметрами, как приведенные в табл. 2, 3. Сегодня на рынке представлена большая гамма дешевых генераторов изображений или псевдодорогих, для которых большинство параметров, рассмотренныхв статье, совсем не специфицировано, и установки по ним не проходят аттестацию при приемо-сдаточных 


\section{(П) КБТЭМ-ОМО}

ОБОРУДОВАНИЕ ДЛЯ МИКРОЭЛЕКТРОНИКИ

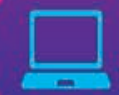

ПРОЕКТИРОВАНИЕ

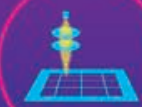

ГЕНЕРАЦИЯ ИЗОБРАЖЕНИЙ

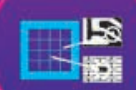

КОНТРОЛЬ ФОТОШАБЛОНОВ

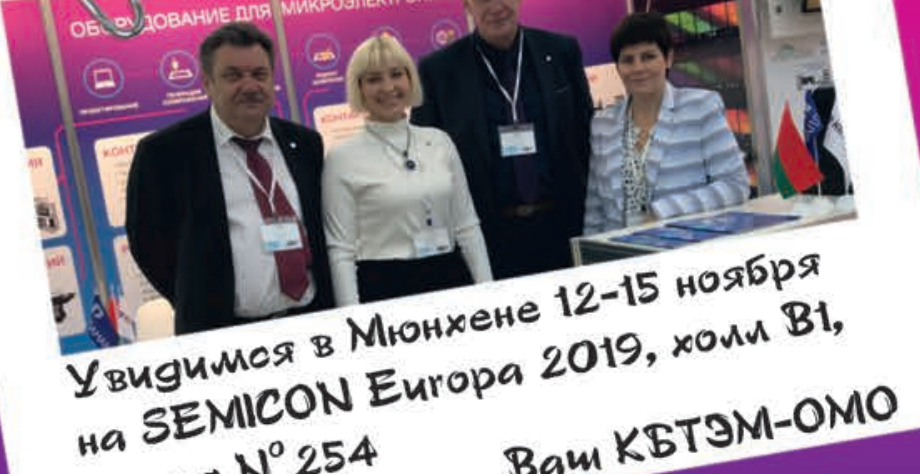

() $\mathrm{s}$.

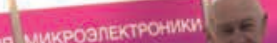

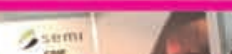

Ha

SEMICON

omeHg
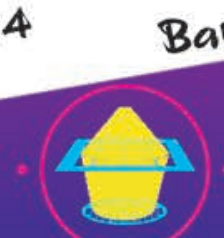

PEMOHT ФОТОШАБЛОНОВ
ФОТОЛИТОГРАФИЯ

КОНТРОЛЬ ПЛАСТИН

БЕЗМАСОЧНАЯ ЛИТОГРАФИЯ

- Многоканальные лазерные

генераторы изображений

- Проектная норма 0.35, $0.6 \mu \mathrm{M}$

- Высокая точность совмещения

- $\oslash 200,150,100$ мM

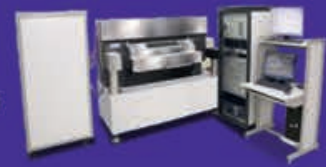

\section{КОНТАКТНАЯ ЛИТОГРАФИЯ}

- Ручная и автоматизированная загрузка

- Двусторонняя литография

- Высокая точность совмещения

- Низкий уровень генерации дефектов

- Высокая энергоэффективность

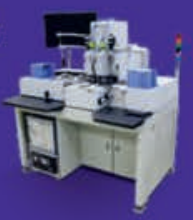

\section{СТЕППЕРЫ}

- Проектная норма 0.35, $0.8 \mu \mathrm{M}$

- Автоматический масштаб

- Двустороннее совмещение

- $\emptyset 100,150,200$ мм

- Твердотельный источник света

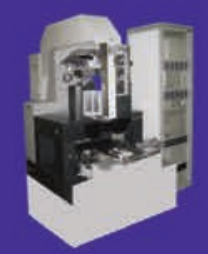

- Быстрая переналадка

пластина - шаблон

КОНТРОЛЬ ФОТОШАБЛОНОВ

- Проектная норма 90,130, 250 нм

- Твердотельный лазер

- Контроль методом Die-to-DB,

Die-to-Die

- Высокая производительность

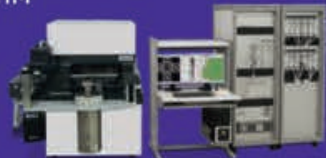

- Контроль неплоскостности

\section{РЕМОНТ ФОТОШАБЛОНОВ}

- Фемтосекундный лазер

- 0.15/ 0.3/ 0.5 нм min элемент

- Размер шаблона до 9"х9"

- Ремонт копированием

- Ремонт через пелликл

- Прозрачные / непрозрачные дефекты

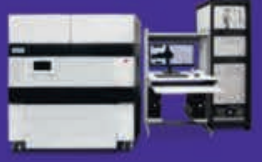

6o

- Единое таможенное пространство

- 58 лет опыта в разработке и производстве прецизионного оптико-механического оборудования

- Высокий уровень применяемых технологий и современного оборудования

- Полный цикл разработки и производства

- Высококвалифицированный персонал

- Высокое качество изделий подтверждено национальными и международными стандартами

- Возможность комплексной поставки оборудования, адаптированного для Российского рынка, программного обеспечения для поддержки процессов изготовления фотошаблонов и 3D-моделирования для фотолитографии компании GenlSys (Германия)

Республика Беларусь

220033, г. Минск

Партизанский пр-т, 2 тел: (+375 17) 2260982

(+375 17) 2237128

факс: (+375 17) 2261205 office@kbtem-omo.by

kbtem.omo@gmail.com

www.kb-omo.by

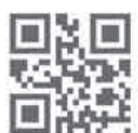


испытаниях. Некоторые параметры хоть и заявлены в спецификации, но их уровень не соответствует уровню технологии, и они к тому же часто не подтверждаются на практике. Например, точность совмещения для технологии 0,6 мкм должна быть нехуже 150 нм, так как этот параметр влияет на величину перекрытия между слоями и в результате определяет размер кристалла. Встречающиеся на рынке установки декларируют точность совмещения 200-1000 нм, хотя никто из пользователей не подтверждает такую точность совмещения и чаще всего сообщает о точности совмещения 1,5-3 мкм, что может устроить только изготовителей очень простых изделий микроэлектроники.

Для контроля большинства параметров генераторов изображений требуется дорогостоящее прецизионное контрольно-измерительное оборудование, которое имеется только у серьезных поставщиков. Контроль таких параметров, как воспроизводимость критического размера, нелинейность элемента топологии, точность совмещения с предыдущим слоем, требуют измерения расстояний в единицы нанометров, что возможно только на хорошем растровом электронном микроскопе (РЭМ), работающем с увеличением не менее 40000 крат. Для контроля точности расположения элемента требуются установки для контроля координат топологии, позволяющие измерять расстояния в сотни миллиметров с точностью несколько нанометров. Измерения такого уровня позволяют реализовать такие уникальные установки, как ЭМ-6209 (КБТЭМ-ОМО) или IPRO (Leica).

\section{ГЕНЕРАТОРЫ ИЗОБРАЖЕНИЙ}

Генератор изображений ЭМ-5589 (рис. 2) - это многолучевой (16 лучей) лазерный генератор изображений, предназначенный для создания топологии по безмасковой технологии с проектными нормами 0,6 мкм и более на полупроводниковых (или др.) подложках. Для экспонирования используется твердотельный лазер, работающий на i-line с гарантированной наработкой на отказ более 22 тыс. часов.

Генератор изображений ЭМ-5289Б (рис. 3) - многолучевой (32 луча) лазерный генератор изображений, предназначенный для создания топологии по безмасковой технологии с проектными нормами 0,35 мкм и более на полупроводниковых (или др.) подложках. Для создания топологии используется газовый лазер, работающий в DUV-диапазоне.

В генераторах изображений загрузка стандартных полупроводниковых пластин проводится автоматически с кассеты. Все функции аттестации и работы генераторов автоматизированы. В генераторах есть встроенные системы пассивной и активной защиты от вибрации. Конструктивные элементы генераторов изображений, отвечающие

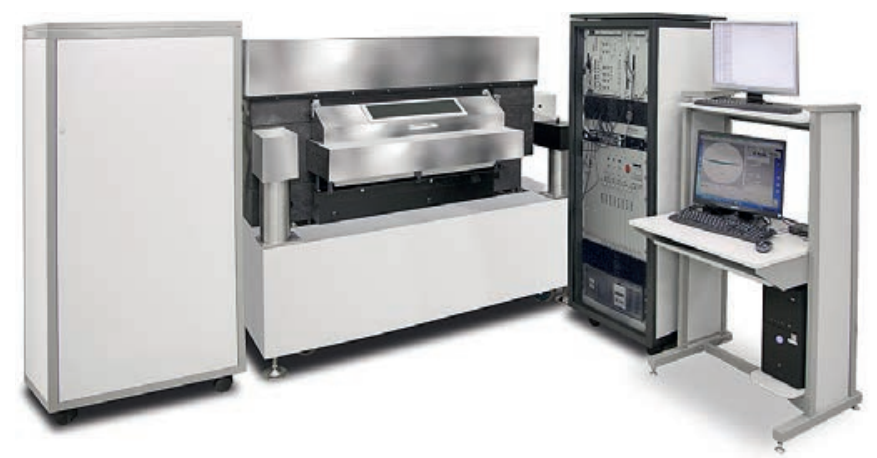

Рис. 2. Внешний вид генератора изображений эМ-5589

за создание топологии, выполнены из термостабильных материалов. Все это в комплексе позволяет обеспечить высокое качество и достоверность создаваемой топологии на полупроводниковых подложках [3]. Более подробную информацию можно получить на нашем сайте (http://kb-omo.by/) или по электронной почте (kbtem.omo@gmail.com).

Представленные в этой статье генераторы изображений производства ОАО «КБТЭМ-ОМО» сегодня успешно применяются для безмасковой литографии в серийном производстве изделий микроэлектроники на передовыхпредприятиях Российской Федерации. Как показала практика, по безмасковой технологии можно изготовить настолько уникальные изделия, которые невозможно было бы создать при помощи другого оборудования.

\section{ЛИТЕРАТУРА}

1. Плебанович В. Создание ЭКБ. Инновационные решения или импортозамещение? // ЭЛЕКТРОНИКА: Наука, Технология, Бизнес. 2015. № 1(00141). С. 110-118.

2. Плебанович В. Безмасковая литография - требование сегодняшнего дня // ЭЛЕКТРОНИКА: Наука, Технология, Бизнес 2015. № 7(00147). С. 112-118.

3. Аваков С. Все ключевые технологии мы реализуем у себя на предприятии // ЭЛЕКТРОНИКА: Наука, Технология, Бизнес. 2018. № 5(00176). С. 12-18. 